\title{
Application of agent-based modelling to assess single-molecule transport across the cell envelope of $E$. coli
}

\author{
Paulo Maia ${ }^{\mathrm{a}}$, Gael Pérez-Rodríguez ${ }^{\mathrm{b}, \mathrm{c}, \mathrm{d}}$, Martín Pérez-Pérez ${ }^{\mathrm{b}, \mathrm{c}, \mathrm{d}}$, Florentino Fdez-Riverola ${ }^{\mathrm{b}, \mathrm{c}, \mathrm{d}}$, \\ Anália Lourenço ${ }^{\mathrm{b}, \mathrm{c}, \mathrm{d}, \mathrm{e}}$, Nuno F. Azevedo ${ }^{\mathrm{a}, *}$ \\ ${ }^{a}$ LEPABE - Dep. of Chemical Engineering, Faculty of Engineering, University of Porto, Rua Dr. Roberto Frias, 4200-465, Porto, Portugal \\ ${ }^{\mathrm{b}}$ ESEI - Dep. of Computer Science, University of Vigo, Edificio Politécnico, Campus Universitario As Lagoas S/n, 32004, Ourense, Spain \\ ${ }^{\mathrm{c}}$ CINBIO - Centro de Investigaciones Biomédicas, University of Vigo, Campus Universitario Lagoas-Marcosende, 36310, Vigo, Spain \\ ${ }^{\mathrm{d}}$ SING Research Group, Galicia Sur Health Research Institute (ISS Galicia Sur), SERGAS-UVIGO, 36312, Vigo, Spain \\ ${ }^{\mathrm{e}}$ CEB - Centre of Biological Engineering, University of Minho, Campus de Gualtar, 4710-057, Braga, Portugal
}

A R T I C L E I N F O

\section{Keywords:}

Modelling

Simulation

Agent-based modelling

Cellular noise

Molecular diffusion

Cell envelope

\begin{abstract}
A B S T R A C T
Motivation: Single cells often show stochastic behaviour and variations in the physiological state of individual cells affect the behaviour observed in cell populations. This may be partially explained by variations in the concentration and spatial location of molecules within and in the vicinity of each cell.

Methods: This paper introduces an agent-based model that represents single-molecule transport through the cellular envelope of Escherichia coli at the micrometre scale. This model enables broader observation of molecular transport throughout the different membrane layers and the study of the effect of molecular concentration in cellular noise. Simulations considered various low molecular weight molecules, i.e. ampicillin, bosentan, coumarin, saquinavir, and terbutaline, and a gradient of molecular concentrations. The model ensured stochasticity in the location of the agents, using diffusing spherical particles with physical dimensions.

Results: Simulation results were validated against theoretical and experimental data. For example, theoretically, ampicillin molecules take $0.6 \mathrm{~s}$ to cross the entire cell envelope, and computational simulations took $0.68 \mathrm{~s}$, $0.68 \mathrm{~s}, 0.70 \mathrm{~s}$, and $0.69 \mathrm{~s}$, for concentrations of $1.44 \mu \mathrm{M}, 13.21 \mu \mathrm{M}, 26.4 \mu \mathrm{M}$ and $105.61 \mu \mathrm{M}$, respectively. Replicate standard deviation decreased with growing initial concentrations of the molecules. In turn, no clear relationship could be observed between molecular size and variability.

Conclusions: This work presented a novel agent-based model to study the effect of the initial concentration of low molecular weight molecules on cellular noise. Cellular noise during molecule diffusion was found to be concentration-dependent and size-independent. The new model holds considerable potential for future, more complex analyses, when emerging experimental data may enable modelling of membrane transport mechanisms.
\end{abstract}

\section{Introduction}

Biological processes in the cell envelope are hard to replicate in vitro because the native protein-lipid architectures and dynamics in the membrane environment are poorly characterized [1,2], and the complex protein network, which is fundamental for molecule exchange, is hard to mimic in laboratory conditions [3]. Furthermore, the use of molecular fluorescent labels for single particle tracking revealed that the biological processes occurring within the cell are often stochastic $[4,5]$, resulting in differences between in vivo and in vitro experiments [6]. These differences may be caused by cellular noise, which is generally described as an irregular temporal fluctuation (noise process) on the physiological state of the cells of a population with no genetic variability and exposed to a similar microenvironment $[7,8]$.

As an alternative, the construction and simulation of in silico molecular models can help understand cell envelope processes and cellular behaviour [9]. Specifically, agent-based models (ABM) have shown great potential to simulate biological events [10-14]. Typically, these models represent the cell as a complex environment, where single molecules are described as individual agents that are influenced by their surroundings. As such, the behaviour of each molecule can be understood by conducting simulations that (as far as possible) mimic the conditions of in vivo systems. Notably, the computational simulation of the spatial location, diffusion and interaction of molecules (included

\footnotetext{
${ }^{*}$ Corresponding author.

E-mail addresses: up201406159@fe.up.pt (P. Maia), gprodriguez2@esei.uvigo.es (G. Pérez-Rodríguez), mpperez3@esei.uvigo.es (M. Pérez-Pérez), riverola@uvigo.es (F. Fdez-Riverola), analia@uvigo.es (A. Lourenço), nazevedo@fe.up.pt (N.F. Azevedo).
} 
in the definition of the agents and agent events) in three-dimensional, continuous environments enables the reproduction of the cellular noise related to spatiotemporal variation [15].

Therefore, this work proposes the application of agent-based modelling to the realistic, three-dimensional simulation of the diffusion of small molecules throughout the different layers of the cellular envelope of Escherichia coli. Arguably, the diffusion or transport of low molecular weight (LMW) molecules is one of the most important biological processes involving the cell envelope of bacteria. Processes involving LMWs transport are not only essential for the survival of the microorganisms [16] but also crucial to develop antibiotics that must cross the cellular envelope in order to be effective [17].

Therefore, the new model includes strategies to adjust and validate simulation parameters against experimental data or, in their absence, theoretical values (e.g. the classical theoretical calculus of moleculespecific diffusion coefficients for each layer of the cell). The main goal of this work was to study the effect of the initial concentration of LMW molecules on cellular noise. The molecules ampicillin, bosentan, coumarin, saquinavir, and terbutaline were selected as case studies based on the availability of experimental data and differences in molecular weight.

\section{Methods}

The following sections describe the computational modelling of single-molecule transport through the $E$. coli cell envelope and, most notably, the experimental data and biological assumptions that supported the construction of such model.

Generally, the new ABM is composed of a set of agents that represent the cell membranes and a population of agents, which represents the molecules under study. Agent characterisation comprises a geometric shape, dimensions (including thickness), diffusion rates (including permeability coefficients), and behavioural logic. Biomolecular events unfold on an explicit and specific environment (i.e. representing the cell and some of the surrounding extracellular space) where agents act autonomously, executing individual paths (i.e. molecular diffusion). Agents interact with one another following common biochemical and biophysical assumptions, and their behaviour is influenced by the immediate surroundings (e.g. transport through a layer).

Table 1 describes the representation of the environment and the agents in the ABM according to the experimental data currently available about the transport of LMW molecules through the cell envelope of E. coli. When available, experimental data was applied. Otherwise, theoretical approximations based on well-established biological assumptions were used.

The model simulations were conducted in the Multi-Agent Simulator Of Neighborhoods (MASON) framework [18]. The rate of conversion from simulation time (i.e. timesteps) to biological time was $2.05 \times 10^{9}$ timesteps/s, as previously described in other work from our group [11]. For simplicity, all results are presented in biological time. See Supplementary material 1 for more detailed information about the experimental and mathematical assumptions applied to model parametrisation.

\subsection{Environment: dimensions and structure of $E$. coli}

The proposed ABM considers the passive transport of small molecules in the cellular envelope of $E$. coli, i.e. a model organism that has available a comprehensive set of experimental data. The rod-shaped $E$. coli cell was implemented as a spherocylinder with a radius ( $r$ ) of $380 \mathrm{~nm}$ and a height (h) of $1940 \mathrm{~nm}$. Moreover, the different layers of the cell envelope of this Gram-negative bacterium (including the peptidoglycan layer) were individually characterized (Table 2).

Cell dimensions are highly dependent on the growth conditions, as observed by the wide range of experimental values retrieved from the scientific literature. Simulations used the lowest values of cell radius

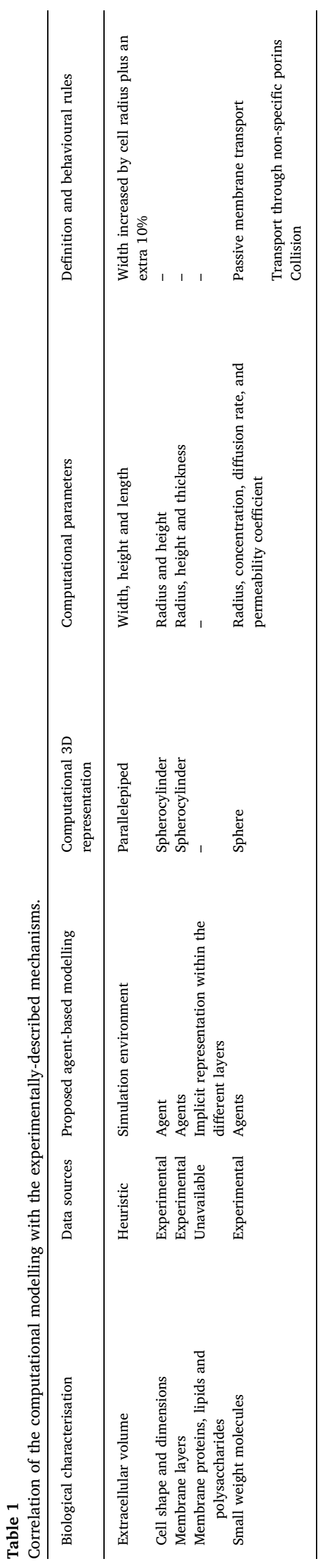


Table 2

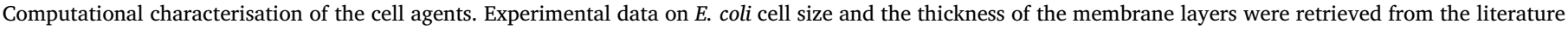
and further applied in model simulation.

\begin{tabular}{|c|c|c|c|}
\hline Parameter & Experimental value range $(\mathrm{nm})$ & Reference & Simulation value (nm) \\
\hline Cell radius $[\mathrm{r}]$ & $380 \pm 20$ & [19] & 380 \\
\hline Cell height $[\mathrm{h}]$ & 1,94 to 2,72 & {$[20]$} & 1940 \\
\hline Inner membrane thickness & $3.75 \pm 0.05$ & {$[21]$} & 3.8 \\
\hline Outer membrane thickness & $13 \pm 1.0$ & {$[22]$} & 13 \\
\hline Peptidoglycan thickness & $6.35 \pm 0.53$ & {$[23]$} & 6.4 \\
\hline Periplasm thickness & 11 to 15 & [24] & 13 \\
\hline Capsule thickness & 2 to 8 & {$[25]$} & $0^{\mathrm{a}}$ \\
\hline
\end{tabular}

a Since not every bacterium has a capsule, and there is very little data regarding diffusion through it, the existence of the capsule was not considered.

and height as these were considered to better reflect the nutrient-limitation that $E$. coli finds in most situations. The thickness of each layer was set based on the average of available experimental values. In theory, a lipid bilayer has a thickness of around $4 \mathrm{~nm}$ [26]. However, and most likely due to the extra constituents of the outer membrane, such as proteins and lipopolysaccharides [27], the outer membrane is generally considered to be thicker than the cytoplasmic membrane.

The model also considers the extracellular volume from where the molecules would approach the cell. To avoid unnecessary computation, the model represented a sub-volume of the $E$. coli cell and extracellular space of $0.23 \mu \mathrm{m}^{3}$. For this volume, the number of agents never exceeded 25,000, a number that allows computation time to take less than a day. Table 3 describes the dimensions of the simulated environment. Furthermore, the representation of obstacles, i.e. mobile but non-reactive agents, was implemented to approximate the volumetric composition of the $E$. coli cytoplasm and thus, be able to evaluate the impact of molecular crowding [28-30].

\subsection{Molecular agents and behavioural rules}

Experimental data on the molecules ampicillin, bosentan, coumarin, saquinavir and terbutaline were used to validate the proposed ABM. All these molecules are aromatic organic chemical compounds for which molecular formula and chemical structures are known. For most of them, there is no evidence of active or facilitated transport mechanisms through the E. coli cell envelope. Bosentan, coumarin, saquinavir and terbutaline are known to permeate directly through lipid bilayers [31]. Ampicillin is known to permeate through a porin (OmpF), apparently by a spontaneous passive diffusion process [32].

Due to the lack of information about the permeability coefficients in the literature, the main factor for the selection of these molecules was the availability of experimentally-calculated values for the permeability coefficients through lipid bilayers (i.e. the values for bosentan, coumarin, saquinavir and terbutaline were obtained for Caco-2 cells) and the porin that crosses the external lipid bilayer (i.e. the value for ampicillin was obtained for $E$. coli). These data enabled the realistic characterisation of each molecular volume, the diffusion rates in the exterior of the cell $\left(D_{o}\right)$ and the periplasm $\left(D_{\text {peri }}\right)$, as well as the permeability in lipid membranes (Table 4). The theoretical hydrodynamic radii and the diffusion coefficient of the molecules were calculated based on classical theories, as described in a previous work [33] (see details in sections 2 to 6 of supplementary material 1 ).

At the start of the simulation, the concentration of molecule agents are randomly distributed outside the cell, moving according to the corresponding $D_{o}$, with an initial random three-dimensional orientation. When these agents collide with the cell envelope, their diffusion rate is adjusted according to the permeability in the outer membrane. Likewise, while traversing the different membrane layers, the diffusion rate of the agents is re-adjusted according to the characteristics of the traversed layer. Specifically, such rate adjustment is applied when the agent has already diffused through a volume corresponding to half of its radius of interaction.

\subsection{Statistical analysis}

Each simulation included six replicates. The average first-passage time of the molecules through the beginning of each layer was calculated. These results were analysed to observe if there were any statistically significant differences between simulation results and the theoretical values for diffusion through each layer (deduced mathematically using Eq. 2 to 5 in supplementary material 1). The one-sample Wilcoxon test, a non-parametric alternative test to the one-sample $t$ test, supported this analysis. The goal was to compare a measure of central tendency of the population under observation (i.e. the median) with a given theoretical value. This test was considered adequate because the sample size was small and this test does not require the population to be normally distributed. The significance level was set to $5 \%$ $(\mathrm{P}<0.05)$.

\section{Results}

Fig. 1 summarises the main computational features of the proposed agent-based model and the data supporting computational simulation. The proposed model was specified in a JSON file compliant with the MASON ABM framework, which enabled model simulation, including real-time graphical visualisation and the generation of detailed results about the position and velocity of the individual agents throughout the simulation.

Initial simulations aimed to calibrate agent velocity in individual layers. The average time taken by the agents to cross each individual layer was calculated based on the simulation results, i.e. the position of the individual agents in the simulation. Agent velocity was then

Table 3

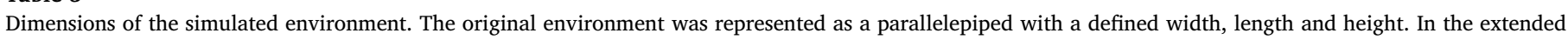

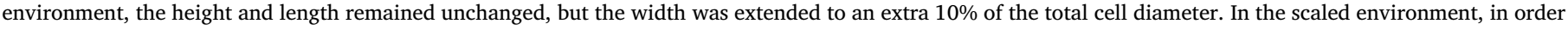

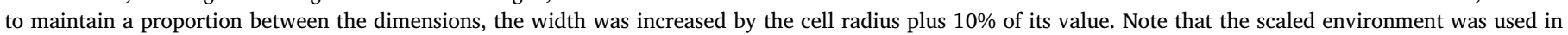
order to reduce computational time, and the scaling percentage was determined experimentally.

\begin{tabular}{|c|c|c|c|}
\hline Dimension of simulation environment & Original environment & Extended width environment & Scaled environment \\
\hline WIDTH (MM) & 2.752 & 2.827 & 0.414 \\
\hline HEIGHT (MM) & 0.752 & 0.752 & 0.752 \\
\hline LENGTH (MM) & 0.752 & 0.752 & 0.752 \\
\hline
\end{tabular}


Table 4

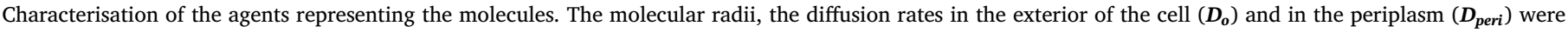

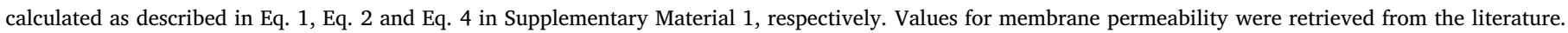

\begin{tabular}{|c|c|c|c|c|c|}
\hline Molecules $^{\mathrm{a}}$ & Molecular weight (Da) & Radius (nm) & $\mathbf{D}_{\mathbf{o}}\left(\times \mathrm{E}^{-10} \mathrm{~m}^{2} / \mathrm{s}\right)$ & $\mathbf{D}_{\text {peri }}\left(\times \mathrm{E}^{-10} \mathrm{~m}^{2} / \mathrm{s}\right)$ & Membrane permeability $(\mu \mathrm{m} / \mathrm{s})$ \\
\hline Ampicillin & 349.41 & 0.423 & 7.75 & 3.13 & $0.028[32]$ \\
\hline Bosentan & 551.62 & 0.485 & 6.77 & 2.56 & $1.05 \times 10^{-2}[31]$ \\
\hline Coumarin & 146.14 & 0.307 & 10.07 & 4.96 & $0.776[31]$ \\
\hline Saquinavir & 670.85 & 0.537 & 6.11 & 2.19 & $5.50 \times 10^{-3}[31]$ \\
\hline Terbutaline & 222.31 & 0.376 & 8.73 & 3.73 & $3.80 \times 10^{-3}[31]$ \\
\hline
\end{tabular}

a The initial agent concentration on the exterior of the cell was $26.4 \mathrm{mM}$.

adjusted iteratively until it was similar to the theoretical value. Table 5 displays the results of agent velocity calibration for membrane layers. Noteworthy, the average time taken to transverse the membrane layers is very similar to the values calculated previously using the theoretical algebraic models.

Following simulations assessed the diffusion of the studied molecules for the entire cell envelope. Fig. 2 shows the simulation results for the five molecules at each of the membrane layers, considering an initial concentration of $26.4 \mu \mathrm{M}$ (i.e. 3657 agents). As expected, the smaller agents are the fastest, irrespective of the layer where the diffusion is occurring, with the exception of terbutaline that has a slower movement in the outer and inner membrane. This was expected, as the permeability coefficient retrieved from the literature for this molecule was smaller than the permeability coefficient for the other molecules [31]. It is well known that permeability coefficients are affected not only by the molecular size but also by the interactions established between the molecule and the components of the membrane. Statistical results (Supplementary material 2) show that the difference between the simulated and the theoretical results is statistically significant in some of the cases, such as for the diffusion of bosentan in the periplasm and the inner membrane $(\mathrm{P}<0.05)$. Differences in these cases may be explained by the fact that the calibration performed for each of the individual layers is iterative and stops when the percentage of difference is below a pre-defined threshold. Nonetheless, all differences were typically below $6 \%$, meaning that the final results were unlikely to be very affected by these deviations.

Fig. 3 details these simulation results for ampicillin. Overall, the theoretical data fit well with the simulation data, with average differences ranging from $1.74 \%$ (for diffusion in the inner membrane) to $4.64 \%$ (for diffusion in the periplasm). Only the difference between the theoretical and simulation values of periplasm diffusion was statistically significant $(P<0.05)$, and this was justified by the reasons aforementioned. It was possible to observe that: $(i)$ on average, an ampicillin agent took $0.52 \mathrm{~s}$ to cross the outer membrane, the fastest ampicillin molecule took only $0.2 \mathrm{~s}$, and the slowest molecule took $7.91 \mathrm{~s}$; (ii) the fastest molecule took $0.27 \mathrm{~s}$ to cross the entire cell envelope, whereas the slowest molecule took $8.07 \mathrm{~s}$.

Moreover, the ratio obtained for the outer membrane and inner membrane diffusion times of ampicillin was around 3.7 , while the ratio for these layers' thickness is 3.4. This occurred because no physical difference between the layers was considered (i.e. diffusional properties were the same for both membranes), and the different diffusion times were only related to their different thicknesses. Supplementary material 2 provides detailed data on the rest of simulations, supplementary material 3 shows snapshots of these simulations, and supplementary material 4 provides a video of the simulation of ampicillin diffusion throughout the cell envelope.

Next, simulations were conducted to evaluate the impact of molecule concentration and molecular weight in cellular noise. Fig. 4 and supplementary material 5 illustrate the simulation results obtained for the diffusion of the five molecules through all the layers of the cell envelope, considering four different initial concentrations. Fig. 4 shows the direct relationship between the standard deviation of the total time that takes a given molecule (at a given concentration) to go through the cellular envelope. On supplementary material 5, boxplots represent the variability observed, and the dashed lines indicate the corresponding theoretical value. As an example, one can observe that (i) for the studied concentrations (1.44 $\mu \mathrm{M}, 13.21 \mu \mathrm{M}, 26.4 \mu \mathrm{M}$ and $105.61 \mu \mathrm{M})$, ampicillin molecules took on average $0.68 \mathrm{~s}, 0.68 \mathrm{~s}, 0.70 \mathrm{~s}$, and $0.69 \mathrm{~s}$ to cross the entire cell envelope, respectively; (ii) the theoretical time for the diffusion of ampicillin was approximately $0.6 \mathrm{~s}$. Statistical results (Supplementary material 6) show that the difference between the simulated and the theoretical results is statistically significant in some of the cases, and the percentage difference between the two situations is below $20 \%$. This difference was mainly due to the cumulative deviations that were observed for each of the layers of the membrane.

Simulation results for lower concentrations were more variable. Cellular noise, seen here as the standard deviation in diffusion time, decreased as molecular concentration increased, i.e. standard deviation decreased with growing concentrations. In particular, for any of the observed molecules, it was smaller for the highest concentration (i.e. $105.61 \mu \mathrm{M}$ ) than for the lowest concentration (i.e. $1.44 \mu \mathrm{M}$ ). Moreover, there was no clear relationship between molecule size and cellular noise. For instance, for a concentration of $1.44 \mu \mathrm{M}$, the smallest molecule, i.e. coumarin, showed a higher standard deviation than ampicillin, which had a higher radius. On the other hand, saquinavir, which was larger than both molecules, had a higher standard deviation than ampicillin. The average total time that a molecule took to go through the cellular envelope was very similar for all concentrations (supplementary material 5). That is, there was also no relationship between molecular concentration and size.

Besides estimating the average deviation for various simulation replicas, the level of variability related to diffusion was inspected based on the estimation of the maximum total time difference $\left(t_{\max }\right)$ for each molecule and concentration. For example, in the case of ampicillin, $t_{\max }$ was lowest for the highest concentration (i.e. 2.17E-03 s for $105.61 \mu \mathrm{M}$ ) and highest for a concentration of $1.44 \mu \mathrm{M}$ (i.e. 2.32E-02 s). In general, a decrease in the concentration leads to an increase in the $t_{\text {max }}$, which is in agreement with the results found for ABM modelling in enzymatic reactions. These results can be generalized to any other molecules with low molecular size to diffuse freely through the periplasm and cross the peptidoglycan layer (see supplementary material 6).

\section{Discussion}

This work introduced a novel ABM to study the effect of the initial concentration of LMW molecules on cellular noise. First, the simulations were validated against experimental values, which indicated the average first-passage time that specific molecules take to cross the individual layers of the envelope, from the outer membrane till the inner membrane. In general, the simulation results agreed well with the theoretical values, but some deviations were to be expected as the conditions of the calibration were not the same as those of the simulations. Also, some variation between simulations was to be expected, because diffusion is a stochastic process, and there are various interactions of the surrounding particles and the medium in each simulation, leading to each particle going a different path. Besides, the initial, random position of the particles in different simulations may lead to 


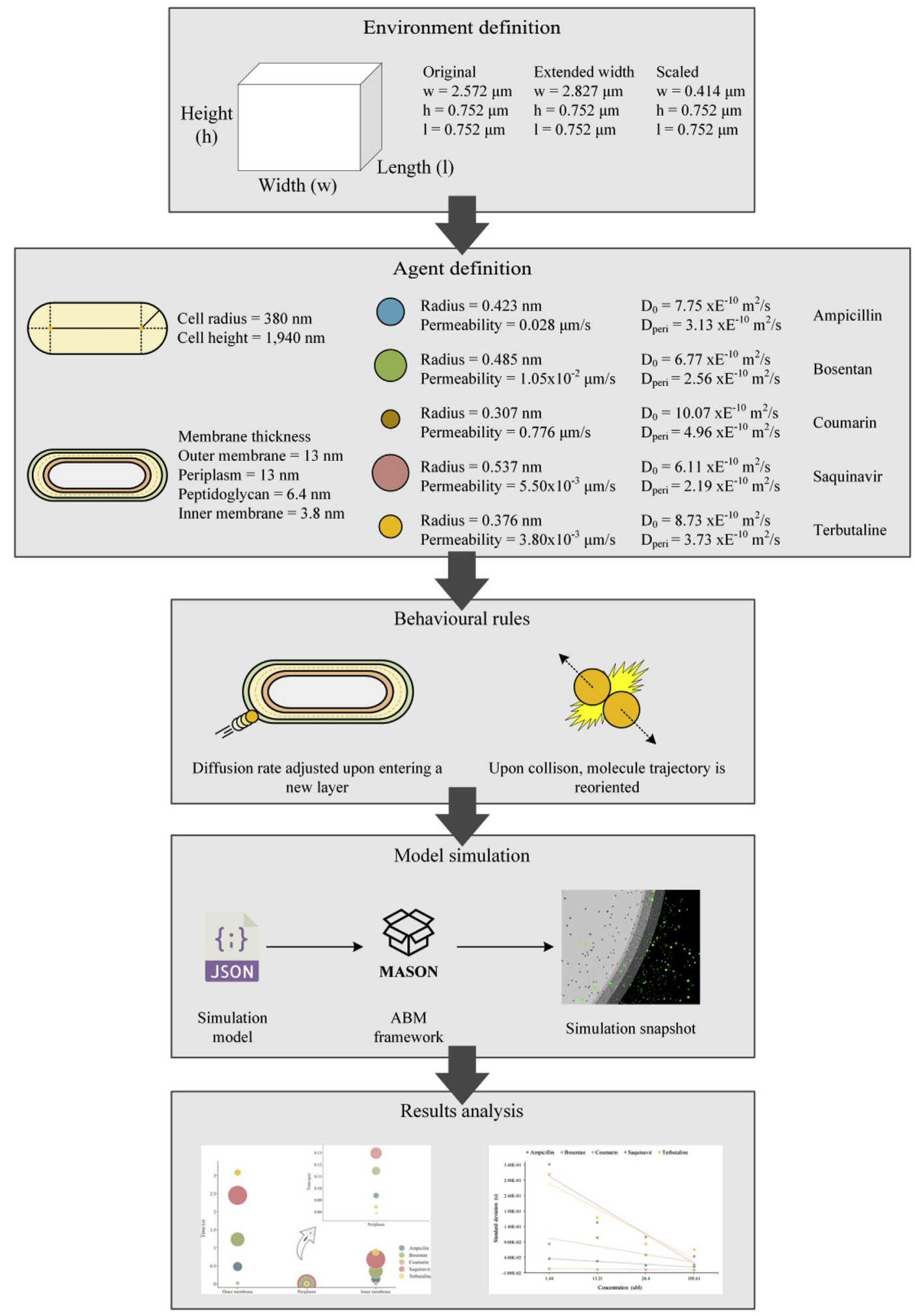

Fig. 1. Main computational aspects of the proposed agent-based model. The simulation environment is defined. Then, the size, shape, thickness, diffusion and permeability of the agents (i.e. E. coli cell, its membrane layers, and the molecules) are specified. After that, the behaviour of the agents is characterized, namely membrane transport and molecule collision. These data are stored in a JSON file that it is used by the MASON framework to simulate the proposed ABM.

different diffusion times.

In order to conduct the proposed simulations, it was necessary to make some assumptions, because our experimental knowledge about diffusional processes in the cellular envelope is still limited, and the computational costs inherent to the simulation of such complex biological scenarios are high $[9,34]$. For instance, the permeability coefficient for ampicillin was obtained for the passive diffusion of small hydrophobic antibiotics through non-specific porins [32]. While porins were not explicitly included in the proposed model, the agent trajectory was adjusted in order to be quite similar to diffusion through a porin. In addition, the diffusion in the inner and outer membranes of Gram-negative cells was considered similar. This is, of course, a simplification, 
Table 5

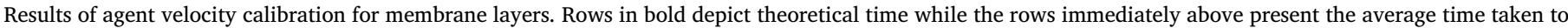
cross the membrane layers in the simulations.

\begin{tabular}{|c|c|c|c|c|}
\hline & \multicolumn{4}{|c|}{ Average time to cross the membrane layer (s) during simulation } \\
\hline & Diffusion rate in the extracellular space $\left(\mu \mathrm{m}^{\wedge} 2 / \mathrm{s}\right)^{\mathrm{a}}$ & Outer membrane & Periplasm & Inner membrane \\
\hline \multirow[t]{2}{*}{ Ampicillin } & $6.9 \mathrm{E}+02$ & $4.5 \mathrm{E}-01 \pm 1.3 \mathrm{E}-02$ & $9 \mathrm{E}-08 \pm 2.5 \mathrm{E}-09$ & $1.4 \mathrm{E}-01 \pm 4.4 \mathrm{E}-03$ \\
\hline & $6.7 E+02$ & 4.6E-01 & $9 \mathrm{E}-08$ & $7.4 \mathrm{E}+00$ \\
\hline \multirow[t]{2}{*}{ Bosentan } & $5.7 \mathrm{E}+02$ & $1.2 \mathrm{E}+00 \pm 5.2 \mathrm{E}-02$ & $1.2 \mathrm{E}+00 \pm 3 \mathrm{E}-02$ & $3.4 \mathrm{E}-01 \pm 3.7 \mathrm{E}-03$ \\
\hline & $5.7 E+02$ & $1.2 \mathrm{E}+00$ & $1.2 \mathrm{E}+00$ & $3.6 \mathrm{E}-01$ \\
\hline \multirow[t]{2}{*}{ Coumarin } & $9.8 \mathrm{E}+02$ & $1.7 \mathrm{E}-02 \pm 5.0 \mathrm{E}-04$ & $6.6 \mathrm{E}-08 \pm 1.6 \mathrm{E}-09$ & $4.9 \mathrm{E}-03 \pm 2.9 \mathrm{E}-04$ \\
\hline & $9.7 \mathrm{E}+02$ & $1.7 \mathrm{E}-02$ & $6.1 \mathrm{E}-08$ & $4.9 \mathrm{E}-03$ \\
\hline \multirow[t]{2}{*}{ Saquinavir } & $4.8 \mathrm{E}+02$ & $2.4 \mathrm{E}+00 \pm 7.5 \mathrm{E}-02$ & $1.4 \mathrm{E}-07 \pm 6.6 \mathrm{E}-09$ & $6.7 \mathrm{E}-01 \pm 1.3 \mathrm{E}-02$ \\
\hline & $5 E+02$ & $2.4+\mathrm{E} 00$ & $1.3 \mathrm{E}-07$ & $6.9 \mathrm{E}-01$ \\
\hline \multirow[t]{2}{*}{ Terbutaline } & $8 \mathrm{E}+02$ & $3.1 \mathrm{E}+00 \pm 4.8 \mathrm{E}-02$ & $7.9 \mathrm{E}-08 \pm 4.1 \mathrm{E}-09$ & $8.9 \mathrm{E}-01 \pm 8.2 \mathrm{E}-03$ \\
\hline & $8 \mathrm{E}+02$ & $3.4 \mathrm{E}+00$ & 7.5E-08 & $1 E+00$ \\
\hline
\end{tabular}

a The time taken by agents to reach the outer membrane depends on the initial localization of the agents and the diffusion rate.

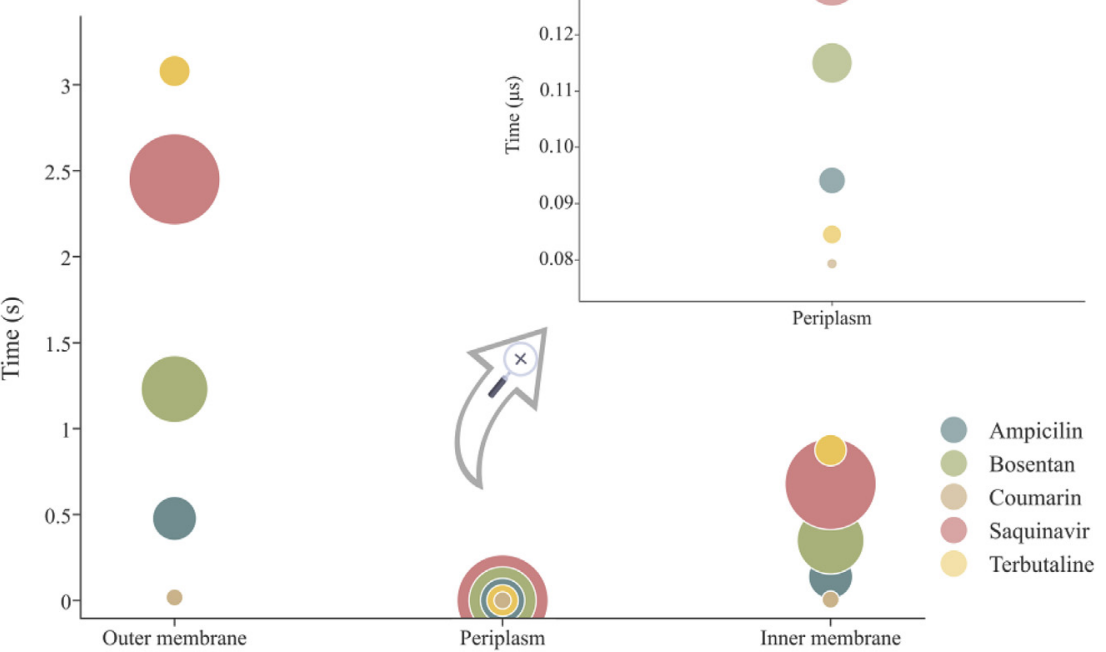

Fig. 2. Simulation results for the five molecules and three layers, representing the experimental diffusion time through each layer, for all 5 molecules. The colour of the disk identifies the molecule and the size of the disk is proportional to the molecular weight.

as it is well-known that the presence of different molecules at different concentrations in the different membranes, such as membrane proteins, lipids and polysaccharides, will affect molecular permeability. Also, due to its composition, it is known that the outer membrane is less susceptible to antibiotic permeation than the inner membrane [35].
Therefore, the absolute times that individual molecules take to cross individual barriers, as calculated here, should be looked at cautiously, as they may be affected by a more realistic representation of the membrane. Nonetheless, the overall conclusion that lower concentrations lead to increased cellular noise is robust, as it is expected that the
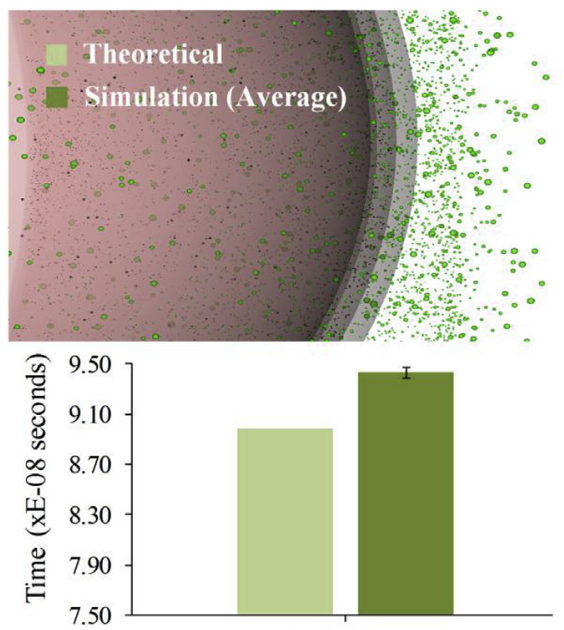

Periplasm
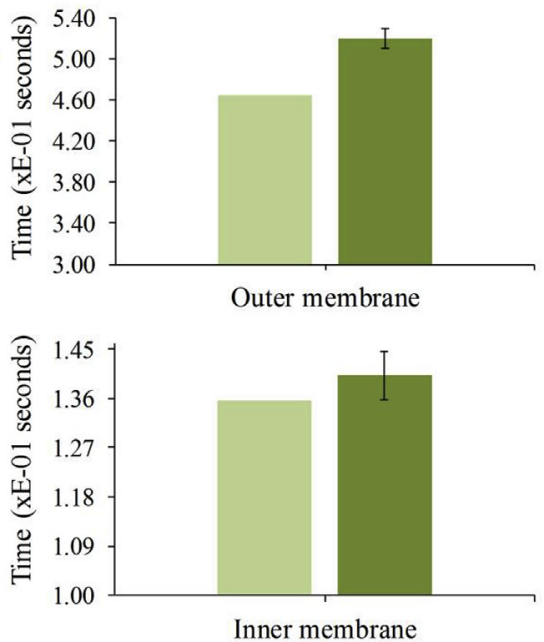

Fig. 3. Comparison of simulation and theoretical results obtained for ampicillin. For the outer and inner membrane, the theoretical diffusion time through each membrane is calculated using Eq. 5 in Supplementary Material 1, while Eq. 3 and 4 were applied to calculate the periplasm value. Green coloured agents represent ampicillin molecules while black coloured agents represent obstacles. The size of the agents is inversely proportional to their distance to the plane shown. The plots show the time taken to cross the entire cellular envelope. Light and dark green bars and black lines represent theoretical and the average of simulation results and the standard deviation of 3 simulation replicates, respectively. 


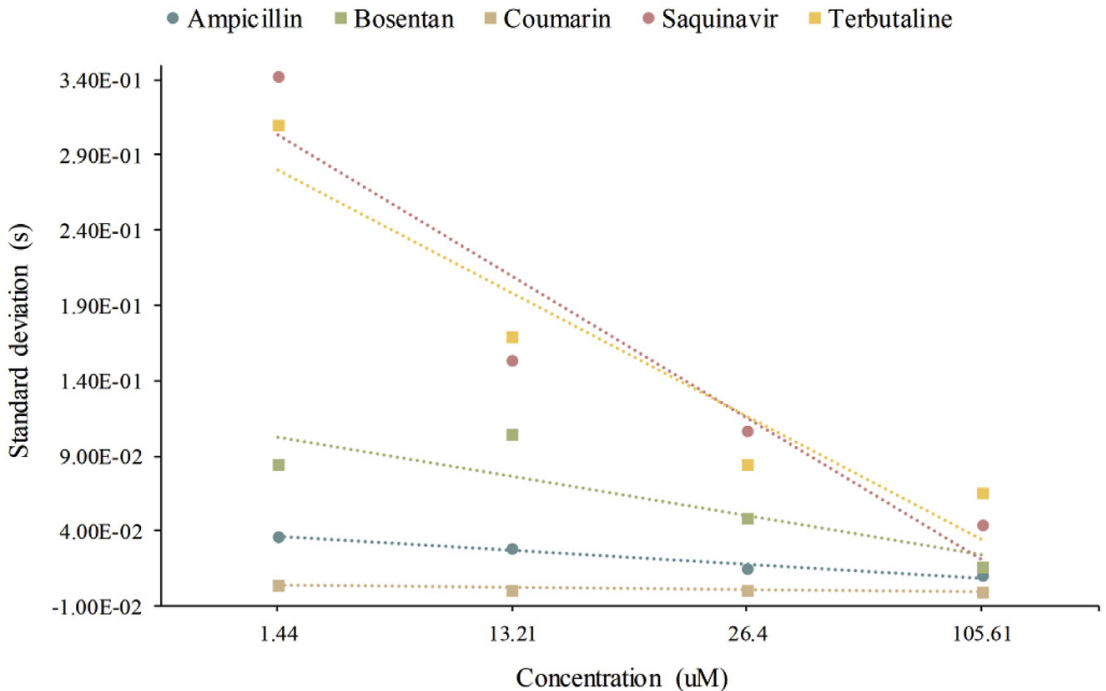

Fig. 4. Comparison of the standard deviation of the total diffusion time of the molecules, considering 6 replicates and increasing concentrations. The concentration referred on the $\mathrm{x}$-axis is the initial concentration on the exterior of the cell, for each agent. In the surrounding layers, the concentration is $0 \mu \mathrm{M}$. On the y-axis, the standard deviation against the theoretical time is given. Dashed lines indicate the trend tendency values for each molecule. Each dot stands for the average of the six replicates obtained for each molecule and concentration. presence of membrane proteins will affect all simulations in a similar manner, regardless of the initial agent concentration.

In order to describe a crowded environment, a simplified approach considering that each molecule had an apparent viscosity was used. Future work using a sdVRC built by adding non-specific interactions may allow a more correct estimation of effective viscosity and diffusion coefficient values in crowded environments [36]. Regarding particle size and shape, it is noteworthy that the Stokes-Einstein equation (Eq. 2 in Supplementary Material 1) is only applicable to spherical molecules [37], although there are corrections for the diffusion of prolate and oblate molecules available [38]. Also important, the equation for crowded diffusion is only valid for $r_{p}$ between $10^{-1}$ and $10^{3} \mathrm{~nm}$, and the curve was built using the van der Waals' radius for amino acids and sugars, and did not include every type of metabolite, so there may be some restrictions when using it for other types of molecules. Recently, it has been shown that the levels of crowding in a cell are heterogeneous, and this model considers the particles to be diffusing through homogeneous environments [39], which can also lead to wrong estimations of diffusion times.

The differences noticed in the time that individual molecules take to cross the cellular envelope can be due to several factors, such as the obstacles that an individual molecule will find in its path - for instance, the molecular crowding in the cytoplasm may delay significantly one individual molecule, whereas another one may have a more straightforward path. Besides, the stochasticity of the diffusion process and the interactions of each molecule with the surrounding environment may also lead to variations in diffusion time. In any case, these differences will arise from the original spatial location and direction of the molecule in the extracellular space. We also observed this previously in ABM studies of reaction kinetics and cellular communication [10,11]. Overall, the spatial location of the agents at the start of the simulation affects, up to a certain extent, the overall process and helps explain why cells have different phenotypes, even when they apparently grow in similar conditions [40].

Even though cellular noise is masked in vitro, because of the limitations of current experimental methods, during in silico experiments individual agents can be tracked throughout time and hence, the heterogeneous movement of particles (that ultimately leads to cellular noise) can be studied [41]. Having this in mind, cellular noise was expected to decrease as the agent concentration increased, and this was indeed observed for all studied molecules. However, no correlation was found between molecule size and cellular noise. Also, for very high drug concentrations, it is possible that we would see differences in the mean diffusion time, due to the increased amount of collisions between molecules. However, for the range of concentrations studied, no differences could be observed.

Currently, the proposed $\mathrm{ABM}$ is able to effectively represent the diffusion of small size molecules through the envelope of a Gram-negative, rod-shaped bacterial cell, with a thin peptidoglycan layer. It is known that other properties of molecules, such as amphiphilic moment, flexibility, globularity and amine steric hindrance affect molecule accumulation and diffusion [35]. These properties can be implemented in future models, although they will likely lead to significant increased computational costs. As more information about the diffusion processes becomes available, the model may be extended to other scenarios [42-44]: for example, to model Gram-positive bacterial cells, which has a thicker peptidoglycan layer [27], or bacteria possessing a cell capsule. This model can also be combined with our previous works $[45,46]$ since the rate of conversion from timestep to real-time is the same. It is also becoming increasingly clear that new experimental technologies, such as single particle tracking, will provide more accurate data and enable model update/extension. Moreover, modelling an environment as having heterogeneous crowding could improve the simulation of cellular noise.

So, the present work may have broader applicability in the future, since it is known that drug resistance is related to cellular noise $[47,48]$. For instance, new models may combine different ABMs to describe the translation process that occurs inside the membrane along with the drug metabolism. Surely these model will need to consider that the crowding effects affect the reaction kinetics [49,50], which could be incorporated using experimental results for the reaction time. By assessing the cellular noise associated with drug uptake and metabolism in an integrated way, these models may help to assess if the initial location of drug molecules (for certain drug concentrations) cause variable responses in seemingly isogenic cells in the same physiological state.

Active and passive transport through ion channels or transporters across the membranes was not modelled here because of the lack of available experimental data. New single particle tracking experiments could be designed to measure the experimental diffusion time, which would give us the characteristics of diffusion of the ensemble of molecules. For modelling passive transport, we could give a specific velocity to a given percentage of the particles undergoing diffusion from the outside to the inside of the cell. For active transport, we could make some particles have a negative velocity after reaching a certain layer of the cell envelope, which would lead them to travel backwards.

\section{Conclusions}

Currently, computational models describing membrane transport 
are either too simplistic or overly specialized [34,51]. Some models represent the cell envelope as a simple space boundary (e.g. locate molecules outside or inside the cell, but not detailing membrane interactions) or provide simple representations of some membrane events (e.g. individual membrane-bound and soluble proteins) [52,53]. In contrast, other works represent very particular, atomically detailed molecular dynamics (e.g. passive membrane permeability) [54-57]. Considering that experimental data on these processes is growing but is still insufficient to produce a fully atomistic detailed model, it is important to create models that are able to characterise molecular transport in the cell envelope at an intermediate scale, i.e. describing membrane layers and molecular dynamics throughout these layers as faithfully as possible.

Herein, a novel ABM was proposed to study the effect of the initial concentration of LMW molecules on cellular noise. Cellular noise during molecule diffusion was found to be concentration-dependent and size-independent. The new model holds considerable potential for future, more complex analyses, when emerging single particle tracking data may enable further modelling of membrane transport mechanisms $[9,56]$. As future work, the ABM could be refactored in order to account for additional parameters, such as the crowding heterogeneity and molecule shape.

\section{Competing interests}

The authors have no competing interests to declare.

\section{Acknowledgements}

This study was supported by the Consellería de Educación, Universidades e Formación Profesional (Xunta de Galicia) under the scope of the strategic funding of ED431C2018/55-GRC Competitive Reference Group, the Portuguese Foundation for Science and Technology (FCT) under the scope of the strategic funding of UID/BIO/ 04469/2019 unit and UID/EQU/00511/2019 units, COMPETE 2020 (POCI-01-0145-FEDER-006939), and NORTE-01-0145-FEDER-000005 - LEPABE-2-ECO-INNOVATION, supported by North Portugal Regional Operational Programme (NORTE 2020), under the Portugal 2020 Partnership Agreement, through the European Regional Development Fund. SING group thanks CITI (Centro de Investigación, Transferencia e Innovación) from the University of Vigo for hosting its IT infrastructure. Also, the authors acknowledge the PhD grants of Martín Pérez-Pérez and Gael Pérez-Rodríguez, funded by the Xunta de Galicia.

\section{Appendix A. Supplementary data}

Supplementary data to this article can be found online at https:// doi.org/10.1016/j.compbiomed.2019.02.020.

\section{References}

[1] F.P. Gao, T.A. Cross, Recent developments in membrane-protein structural genomics, Genome Biol. 6 (2005) 244, https://doi.org/10.1186/gb-2005-6-13-244.

[2] L.M. McMorran, D.J. Brockwell, S.E. Radford, Mechanistic studies of the biogenesis and folding of outer membrane proteins in vitro and in vivo: what have we learned to date? Arch. Biochem. Biophys. (2014), https://doi.org/10.1016/j.abb.2014.02. 011.

[3] J. De Geyter, A. Tsirigotaki, G. Orfanoudaki, V. Zorzini, A. Economou, S. Karamanou, Protein folding in the cell envelope of Escherichia coli, Nat Microbiol. 1 (2016) 16107, https://doi.org/10.1038/nmicrobiol.2016.107 http:// www.nature.com/articles/nmicrobiol2016107\#supplementary-information.

[4] A. Levchenko, I. Nemenman, Cellular noise and information transmission, Curr. Opin. Biotechnol. 28 (2014) 156-164, https://doi.org/10.1016/j.copbio.2014.05. 002.

[5] H. Shen, L.J. Tauzin, R. Baiyasi, W. Wang, N. Moringo, B. Shuang, C.F. Landes, Single particle tracking: from theory to biophysical applications, Chem. Rev. 117 (2017) 7331-7376, https://doi.org/10.1021/acs.chemrev.6b00815.

[6] P. Pfaffelhuber, L. Popovic, Scaling limits of spatial compartment models for chemical reaction networks, Ann. Appl. Probab. 25 (2015) 3162-3208, https://doi. org/10.1214/14-AAP1070.
[7] J.M. Raser, E.K. O'Shea, Noise in gene expression: origins, consequences, and control, Science 309 (2005) 2010-2013, https://doi.org/10.1126/science. 1105891.

[8] B. Snijder, L. Pelkmans, Origins of regulated cell-to-cell variability, Nat. Rev. Mol. Cell Biol. 12 (2011) 119-125, https://doi.org/10.1038/nrm3044.

[9] S. Khalid, N.A. Berglund, D.A. Holdbrook, Y.M. Leung, J. Parkin, The membranes of Gram-negative bacteria: progress in molecular modelling and simulation, Biochem. Soc. Trans. 43 (2015) 162-167.

[10] G. Pérez-Rodríguez, S. Dias, M. Pérez-Pérez, F. Fdez-Riverola, N.F. Azevedo, A. Lourenço, Agent-based model of diffusion of $\mathrm{N}$-acyl homoserine lactones in a multicellular environment of Pseudomonas aeruginosa and Candida albicans, Biofouling 34 (2018) 335-345, https://doi.org/10.1080/08927014.2018.1440392.

[11] G. Pérez-Rodríguez, D. Gameiro, M. Pérez-Pérez, A. Lourenço, N.F. Azevedo, Single molecule simulation of diffusion and enzyme kinetics, J. Phys. Chem. B 120 (2016) 3809-3820, https://doi.org/10.1021/acs.jpcb.5b12544.

[12] D. Gameiro, M. Pérez-Pérez, G. Pérez-Rodríguez, G. Monteiro, N.F. Azevedo, A. Lourenço, Computational resources and strategies to construct single-molecule metabolic models of microbial cells, Brief. Bioinform 17 (2016) 863-876, https:// doi.org/10.1093/bib/bbv096.

[13] F.L. Hellweger, R.J. Clegg, J.R. Clark, C.M. Plugge, J.-U. Kreft, Advancing microbial sciences by individual-based modelling, Nat. Rev. Microbiol. 14 (2016) 461-471, https://doi.org/10.1038/nrmicro.2016.62.

[14] M. Soheilypour, M.R.K. Mofrad, Agent-Based Modeling in Molecular Systems Biology, Bioessays vol. 40, (2018) 1800020, https://doi.org/10.1002/bies. 201800020.

[15] S.M. Stiegelmeyer, M.C. Giddings, Agent-based modeling of competence phenotype switching in Bacillus subtilis, Theor. Biol. Med. Model. 10 (2013) 23, https://doi. org/10.1186/1742-4682-10-23.

[16] N.J. Yang, M.J. Hinner, Getting across the Cell Membrane: an Overview for Small Molecules, Peptides, and Proteins (2015) 29-53, https://doi.org/10.1007/978-14939-2272-7_3.

[17] R.S. Santos, C. Figueiredo, N.F. Azevedo, K. Braeckmans, S.C. De Smedt, Nanomaterials and molecular transporters to overcome the bacterial envelope barrier: towards advanced delivery of antibiotics, Adv. Drug Deliv. Rev. 136-137 (2018) 28-48, https://doi.org/10.1016/J.ADDR.2017.12.010.

[18] S. Luke, C. Cioffi-Revilla, L. Panait, K. Sullivan, G. Balan, MASON, A multiagent simulation environment, Simul. Trans. Soc. Model. Simul. Int. 82 (2005) 517-527, https://doi.org/10.1177/0037549705058073.

[19] S. Bakshi, A. Siryaporn, M. Goulian, J.C. Weisshaar, Superresolution imaging of ribosomes and RNA polymerase in live Escherichia coli cells, Mol. Microbiol. 85 (2012) 21-38, https://doi.org/10.1111/j.1365-2958.2012.08081.x.

[20] N. Grossman, E. a. Ron, C.L. Woldringh, Changes in cell dimensions during amino acid starvation of Escherichia coli, J. Bacteriol. 152 (1982) 35-41.

[21] K. Mitra, I. Ubarretxena-Belandia, T. Taguchi, G. Warren, D.M. Engelman, Modulation of the bilayer thickness of exocytic pathway membranes by membrane proteins rather than cholesterol, Proc. Natl. Acad. Sci. U. S. A 101 (2004) 4083-4088, https://doi.org/10.1073/pnas.0307332101.

[22] M.E. Bayer, Zones of membrane adhesion in the cryofixed envelope of Escherichia coli, J. Struct. Biol. 107 (1991) 268-280 https://doi.org/10.1016/1047-8477(91) 90052-X.

[23] W. Vollmer, S.J. Seligman, Architecture of peptidoglycan: more data and more models, Trends Microbiol. 18 (2010) 59-66, https://doi.org/10.1016/j.tim.2009. 12.004.

[24] L.L. Graham, T.J. Beveridge, N. Nanninga, Periplasmic space and the concept of the periplasm, Trends Biochem. Sci. 16 (1991) 328-329, https://doi.org/10.1016/ 0968-0004(91)90135-I.

[25] F.C. Neidhardt, R. Curtiss, Escherichia coli and Salmonella: Cellular and Molecular Biology, ASM press, Washington, DC, 1999.

[26] G. Van Meer, D.R. Voelker, G.W. Feigenson, Membrane lipids: where they are and how they behave, Nat. Rev. Mol. Cell Biol. 9 (2008) 112-124.

[27] T.J. Silhavy, D. Kahne, S. Walker, The bacterial cell envelope, Cold Spring Harb Perspect. Biol. 2 (2010) a000414.

[28] M.J. Byrne, M.N. Waxham, Y. Kubota, Cellular dynamic simulator: an event driven molecular simulation environment for cellular physiology, Neuroinformatics 8 (2010) 63-82, https://doi.org/10.1007/s12021-010-9066-x.

[29] M.T. Klann, A. Lapin, M. Reuss, Agent-based simulation of reactions in the crowded and structured intracellular environment: influence of mobility and location of the reactants, BMC Syst. Biol. 5 (2011) 71, https://doi.org/10.1186/1752-0509-5-71.

[30] M. Feig, Y. Sugita, Reaching new levels of realism in modeling biological macromolecules in cellular environments, J. Mol. Graph. Model. 45 (2013) 144-156, https://doi.org/10.1016/j.jmgm.2013.08.017.

[31] G. Camenisch, J. Alsenz, H. van de Waterbeemd, G. Folkers, Estimation of permeability by passive diffusion through Caco-2 cell monolayers using the drugs' lipophilicity and molecular weight, Eur. J. Pharm. Sci. 6 (1998) 317-324 http://www. ncbi.nlm.nih.gov/pubmed/9795088, Accessed date: 2 November 2016.

[32] S. Kojima, H. Nikaido, Permeation rates of penicillins indicate that Escherichia coli porins function principally as nonspecific channels, Proc. Natl. Acad. Sci. Unit. States Am. 110 (2013) E2629-E2634.

[33] Y.H. Zhao, M.H. Abraham, A.M. Zissimos, Fast calculation of van der Waals volume as a sum of atomic and bond contributions and its application to drug compounds, J. Org. Chem. 68 (2003) 7368-7373, https://doi.org/10.1021/jo034808o.

[34] A. Boags, P.-C. Hsu, F. Samsudin, P.J. Bond, S. Khalid, Progress in molecular dynamics simulations of gram-negative bacterial cell envelopes, J. Phys. Chem. Lett. (2017) 2513-2518, https://doi.org/10.1021/acs.jpclett.7b00473.

[35] H.I. Zgurskaya, C.A. López, S. Gnanakaran, Permeability barrier of gram-negative cell envelopes and approaches to bypass it, ACS Infect. Dis. 1 (2016) 512-522, 
https://doi.org/10.1021/acsinfecdis.5b00097.

[36] F. Trovato, V. Tozzini, Diffusion within the cytoplasm: a mesoscale model of interacting macromolecules, Biophys. J. 107 (2014) 2579-2591, https://doi.org/10. 1016/j.bpj.2014.09.043.

[37] T. Kalwarczyk, M. Tabaka, R. Holyst, Biologistics-diffusion coefficients for complete proteome of Escherichia coli, Bioinformatics 28 (2012) 2971-2978, https:// doi.org/10.1093/bioinformatics/bts537.

[38] F. Roosen-Runge, M. Hennig, F. Zhang, R.M. Jacobs, M. Sztucki, H. Schober, T. Seydel, F. Schreiber, Protein self-diffusion in crowded solutions, Proc. Natl. Acad. Sci. U. S. A. 108 (2011) 11815-11820, https://doi.org/10.1073/pnas.1107287108.

[39] S. Smith, C. Cianci, R. Grima, Macromolecular crowding directs the motion of small molecules inside cells, J. R. Soc. Interface (2017), https://doi.org/10.1098/rsif. 2017.0047.

[40] M.B. Flegg, S. Rüdiger, R. Erban, Diffusive spatio-temporal noise in a first-passage time model for intracellular calcium release, J. Chem. Phys. 138 (2013) 154103

[41] A. Becskei, B.B. Kaufmann, A. van Oudenaarden, Contributions of low molecule number and chromosomal positioning to stochastic gene expression, Nat. Genet. 37 (2005) 937-944 http://www.nature.com/ng/journal/v37/n9/suppinfo/ng1616 S1.html.

[42] A. Robson, K. Burrage, M.C. Leake, Inferring diffusion in single live cells at the single-molecule level, Philos. Trans. R. Soc. Lond. B Biol. Sci. 368 (2013) 20120029, https://doi.org/10.1098/rstb.2012.0029.

[43] G.I. Mashanov, Single molecule dynamics in a virtual cell: a three-dimensional model that produces simulated fluorescence video-imaging data, J. R. Soc. Interface 11 (2014), https://doi.org/10.1098/rsif.2014.0442.

[44] F. Oswald, E.L.M. Bank, Y.J.M. Bollen, E.J.G. Peterman, Imaging and quantification of trans-membrane protein diffusion in living bacteria, Phys. Chem. Chem. Phys. 16 (2014) 12625-12634, https://doi.org/10.1039/c4cp00299g.

[45] G. Pérez-Rodríguez, D. Gameiro, M. Pérez-Pérez, A. Lourenço, N.F. Azevedo, Single molecule simulation of diffusion and enzyme kinetics, J. Phys. Chem. B 120 (2016) 3809-3820, https://doi.org/10.1021/acs.jpcb.5b12544.

[46] D. Gameiro, M. Pérez-Pérez, G. Pérez-Rodríguez, G. Monteiro, N.F. Azevedo, A. Lourenço, Computational resources and strategies to construct single-molecule metabolic models of microbial cells, Briefings Bioinf. (2015) 1-14, https://doi.org/ 10.1093/bib/bbv096.
[47] J.B. Deris, M. Kim, Z. Zhang, H. Okano, R. Hermsen, A. Groisman, T. Hwa, The innate growth bistability and fitness landscapes of antibiotic-resistant bacteria, Science 80 (2013) 342.

[48] D.A. Charlebois, N. Abdennur, M. Kaern, Gene expression noise facilitates adaptation and drug resistance independently of mutation, Phys. Rev. Lett. 107 (2011), https://doi.org/10.1103/PhysRevLett.107.218101.

[49] R. Grima, S.N. Yaliraki, M. Barahona, Crowding-induced anisotropic transport modulates reaction kinetics in nanoscale porous media, J. Phys. Chem. B (2010), https://doi.org/10.1021/jp9025865.

[50] R. Grima, S. Schnell, A systematic investigation of the rate laws valid in intracellular environments, Biophys. Chem. (2006), https://doi.org/10.1016/j.bpc. 2006.04.019.

[51] J. Parkin, M. Chavent, S. Khalid, Molecular simulations of gram-negative bacterial membranes: a vignette of some recent successes, Biophys. J. 109 (2015) 461-468, https://doi.org/10.1016/j.bpj.2015.06.050.

[52] H. Bai, M.D. Rolfe, W. Jia, S. Coakley, R.K. Poole, J. Green, M. Holcombe, Agentbased modeling of oxygen-responsive transcription factors in Escherichia coli, PLoS Comput. Biol. 10 (2014) e1003595, , https://doi.org/10.1371/journal.pcbi. 1003595.

[53] J. Miller, M. Parker, R.B. Bourret, M.C. Giddings, An agent-based model of signal transduction in bacterial chemotaxis, PLoS One 5 (2010), https://doi.org/10.1371/ journal.pone.0009454.

[54] C.J. Dickson, V. Hornak, R.A. Pearlstein, J.S. Duca, Structure-kinetic relationships of passive membrane permeation from multiscale modeling, J. Am. Chem. Soc. 139 (2017) 442-452, https://doi.org/10.1021/jacs.6b11215.

[55] A. Pavlova, H. Hwang, K. Lundquist, C. Balusek, J.C. Gumbart, Living on the edge: simulations of bacterial outer-membrane proteins, Biochim. Biophys. Acta 1858 (2016) 1753-1759, https://doi.org/10.1016/j.bbamem.2016.01.020.

[56] M. Chavent, A.L. Duncan, M.S. Sansom, Molecular dynamics simulations of membrane proteins and their interactions: from nanoscale to mesoscale, Curr. Opin. Struct. Biol. 40 (2016) 8-16, https://doi.org/10.1016/j.sbi.2016.06.007.

[57] T. Travers, K.J. Wang, C.A. López, S. Gnanakaran, Sequence- and structure-based computational analyses of Gram-negative tripartite efflux pumps in the context of bacterial membranes, Res. Microbiol. 169 (2018) 414-424, https://doi.org/10. 1016/J.RESMIC.2018.01.002. 